\title{
Sets, Set Sizes, and Infinity in Badiou's Being and Event
}

\section{Introduction}

In 2006, Lucca Fraser published the article "The Law of the Subject: Alain Badiou, Luitzen Brouwer and the Kripkean Analysis of Forcing and the Heyting Calculus." 'Still one of the best Anglophone commentaries on Badiou's L'Etre et l'Événement, she argued that Kripke forcing was sufficient to render the theory of the subject in Badiou's L'Être et l'événement within an intuitionistic framework. This pluralization of logical frameworks allowed us some insight into the possibilities of mathematical ontology of which Badiou was not aware. That is, it forced us, and eventually Badiou, to recognize that his commitment to classical logic, where the principle of excluded middle was true, was unnecessary to the claims about the subject within his project. In turn, Badiou agreed and acknowledged this point but argued that this only applied to the subjective dimensions of mathematical ontology. ${ }^{2}$ Classical logic was still needed to express the core of the ontological analysis, based on Zermelo-Frankel set theory with the axiom of choice (hereafter ZFC). ${ }^{3}$

The paper here follows in Fraser's example. Yet instead of dealing with the theory of the subject in L'Être et l'evénement, I turn to the theory of the "count", the fundamental aspect of Badiou's theory of Being and beings in L'Être et l'événement. Further, I am not interested here to challenge the classical logical framework upon which the work is couched, although it is far from unassailable. What is in question here is instead the relation between the count and multiplicity. In particular, I argue that Cantorian transfinite cardinality, a method of reckoning

1 Zachary Luke Fraser (Lucca Fraser), "The Law of the Subject: Alain Badiou, Luitzen Brouwer and the Kripkean Analyses of Forcing and the Heyting Calculus", Cosmos and History: The Journal of Natural and Social Philosophy 2 (1-2/2006), pp. 94-133.

2 Alain Badiou, "New Horizons in Mathematics as a Philosophical Condition: An Interview with Alain Badiou [with Tzuchien Tho]”, Parrhesia 3 (2007), pp. 1-11.

3 Ibid., p. 7. 
the measure of the sizes of sets, is not a necessary feature of the coherence of the project of mathematical ontology in L'Être et l'evénement. The implication of this for Badiou's project is that the "subtraction of the one" also implies a pluralism of the one. That is, the pluralization of how unity is constituted, located, and operational. While subtraction is a rejection of the givenness of the one, the rejection of a view entrenched in traditional metaphysics, it retains the form of the one as the result of the count. Here the one is not a given but a result. From this count, the set theorical universe is populated by the entities generated by the count. However, these entities need not be measured in the standard Cantorian way and can be subject to different forms of measurement.

As will be argued, the implication of the pluralism of the one is that the countas-one of finite and transfinite sets is indifferent to Cantorian cardinality. This indifference is crucial because the count is responsible for the count of the inconsistent multiple at the very heart of the project. This initial count is one that formally introduces the set (within the discourse of ZFC) as the basic term of Badiou's mathematical ontology. This renders questions of measure and, in particular, the measure of the infinite orthogonal to this theory. Badiou emphasizes this in his work, noting that the infinite has no intra-mathematical meaning. Badiou's ontological project is thus not only a subtraction from the metaphysics of the "one" but also a subtraction from the metaphysics of infinity, which is dependent on it. What results instead is a pluralism of these terms based on the more fundamental distinction, as Badiou himself puts it, between concept and existence. ${ }^{4} \mathrm{~A}$ pluralism with respect to the unfolding of the ontological concept is thus no challenge to dimension of existence. This orthogonality reveals that Badiou's ontological project is free to embrace a pluralism about unity and infinity unanticipated by Badiou and the founders of set theory. This pluralism of the one reveals new contours of theoretical possibility previously unexamined by Badiou and his commentators.

\section{Counting and the inscription of the void}

The central goal of Badiou's L'Être et l'événement is to employ the structures provided by set theory (especially ZFC) to analyze existence. What this means is not at all straightforward. Badiou argues neither for a Pythagorean-Platonic ontology in the vulgar sense, where existence reduces to sets, nor mathematical

4 Alain Badiou, Being and Event, trans. O. Feltham, Continuum, London 2005, p. 159. 
reality as ultimate reality, nor for set theory as a model for existence. Rather, no structure, be it mathematical, or something else, could serve as a direct map of ultimate reality since any ontology must first reckon with the fact that Being is itself unstructured. Any ontology must first force Being to relate to a structure that would be alien to it. Hence, for Badiou, since Being is itself unstructured, in order for ontology to be possible, it must be drawn into structure by the means of the count. Hence, this first step into the possibility of a mathematical ontology cannot be given by set theory itself. This "drawing in" is operated by "inscription", the localization of unstructured being into the structure of set theory. Basically, the unstructured is inscribed within structure by the count of the void, since whatever is not already within the structure must appear as, or be a presentation of, a "nothing".

The basic picture then is that Badiou makes the link between set theory and unstructured Being by counting it (Being) as a set containing the void. The positivity of the void in set theory therefore stands in for what is "underneath" structure but nonetheless localized within the set theoretical discourse. This bridge between unstructured Being and the structures of set theory rejects the two traditional tendencies in ontology. The first tendency is that ontology or metaphysics in general is a rational description of the fundamental structures of being. This presumes that Being has an inherent structure that is knowable and reducible to some basic form. We can set this aside because Badiou maintains the view, shared by a lineage of thinkers since the Platonists that Being is inaccessible to us through the means of the categories we apply to beings or the senses. The second notion is that ontology plays the role of drawing out the immanent but obscure qualities of Being-as-such. Badiou similarly rejects this path of analyzing the "deep" allure of the Being by cutting off our relation to the "presence" of Being in favor of reducing it to a count of the void. In short, all that is "above" and "beyond" cannot appear as a "one" (i.e. an entity), therefore it is counted as a "void". This act, which we may term "subtraction", produces nothing mysterious. It is simply the count of the void. This is the null set \{\} or $\emptyset$, the void after it has been counted, that forms the basis of the arithmetic counting of pure sets. From this $\emptyset$, we count its successor $\{\varnothing\}$, and its successor's successor $\{\varnothing,\{\varnothing\}\}$, and $\{\varnothing,\{\varnothing\},\{\varnothing,\{\varnothing\}\}\}$, and so on. The success of this process allows Badiou to provide a key distinction in the mathematical ontology of L'Être et l'événement. That is, the arborescent branching of the count of the null set (the count of the void), allows us to retroactively designate Being, seen through the lens of con- 
sistent structure, as "inconsistent". Hence, instead of subordinating Being to the logic of beings (the unit entity), or searching for a non-rational presence of the Being behind and beyond being, unstructured Being is taken up into ontology (the realm of consistent multiplicity) as "inconsistent multiplicity". This is wholly dependent on the existence of a structurally coherent (and well-ordered) consistent multiplicity.

Badiou's act of ontological subtraction may be seen as deflationary to any metaphysics. It is certainly fair to judge it so from the perspective of the traditional goals of ontology. Yet, it is not deflationary of ontology insofar as the subtraction does not restrict ontology to consistent multiplicity. Instead, the distinction between consistent and inconsistent multiplicity provides the central materials for analysis in L'Être et l'événement. Ontology, in Badiou's sense, has therefore the task of unfolding the unexpected relations between consistent and inconsistent multiplicity. More importantly, the fundamental aim of the book, the claim that truths and events exist, is the claim that, because of the incompleteness to any sufficiently strong set theory, there are sets that can be proven to exist but cannot be constructed (non-constructible sets). Hence, the inscription of Being into set theory through the count of the void will entail the unfolding, albeit incomplete, of the inconsistent multiple within the structure of consistent multiplicity.

From the point of the inscription of Being, qua inconsistent multiplicity, the operation of the count is crucial in distinguishing between ordered and structured presentation (sets in set theory), and inconsistent multiplicity (the inscribed void). In this way, structured presentation, what Badiou calls "consistent multiplicity" is given by the ordered universe of pure sets. With the inscription of the count, further counts produce a structure of sets that are recursive counts of the inscription. We can illustrate this with the sequence of natural numbers (0,1,2,3, etc.).

We can note that this counting structure maps succession order with size. However, this correspondence between order and size will not be so obvious when it comes to counts that go beyond the finite numbers. What is conspicuous here is that Badiou's ontology in L'Être et l'événement relies on the count and therefore ordinality. Cardinality plays a much less important role. Why does this difference matter? It matters insofar as the count cannot be subordinated to the difference between the finite and the non-finite (or infinite). The oneness of 
the set is indifferent to whether the set is the void qua inconsistent multiple, a defined quantity (such as the keys on a qwerty keyboard), or the uncountably many points on a continuum. Badiou's use of set theory to anchor mathematical ontology relies fundamentally on ordinality. We shall underline this claim and its stakes further.

\section{What is the difference between cardinality and ordinality?}

Now, the two concepts of cardinality and ordinality are not determined by the axioms of ZFC. The axioms indicate and restrict the kinds of sets that can exist. Cardinality and ordinality are instead structures we use to analyze these sets in terms of "how many" and "which one", respectively, of a given set. In other words, they measure and count sets, respectively. Cardinality can be determined by matching or mapping (measurement) a set onto another pre-given set (i.e. numbers) or itself while ordinality is determined by ordering. Under normal finitary circumstances, cardinality and ordinality correspond neatly to each other, a set of five marbles has a cardinality of five because the five marbles can be mapped to a set of five entities in a pre-given set or to the first five natural numbers. On the other hand, putting the marbles in order will get us to the "fifth" marble, thereby completing the ordinal count of the marbles. ${ }^{5}$ Given the set of finite natural numbers, which does not terminate, any arbitrarily large number will be finite and the ordinal that counts the sequence that allows us to arrive at that number will also tell us the size (cardinal) of the set that is given. The situation is slightly different for non-finite sets.

For finite cases, we can arrange the natural numbers in order and map them to their subsets (e.g., the squares, cubes and fourths, etc.). This one-to-one mapping is the structure of cardinality that allows us to see that these sets are the same size.

\begin{tabular}{|l|l|l|l|l|l|l|}
\hline Naturals $\mathrm{n}$ & 1 & 2 & 3 & 4 & 5 & $\ldots$ \\
\hline Squares $\mathrm{n}^{2}$ & 1 & 4 & 9 & 16 & 25 & $\ldots$ \\
\hline
\end{tabular}

There are ways to deliberately distinguish cardinality and ordinality in finite sets, exploiting the fact that ordinalities are pertinent to ordering while cardinalities are indifferent to order as we shall later examine. 


\begin{tabular}{|l|l|l|l|l|l|l|}
\hline Cubes $\mathrm{n}^{3}$ & 1 & 8 & 36 & 64 & 125 & $\ldots$ \\
\hline Fourths $\mathrm{n}^{4}$ & 1 & 16 & 81 & 256 & 625 & $\ldots$ \\
\hline
\end{tabular}

This is a principle known at least since Thābit ibn Qurra but better known through Galileo who puts forth this mapping in the first day of the Discourses and Mathematical Demonstrations Relating to Two New Sciences. ${ }^{6}$ The point here is that, through mapping, we can clearly see that the measure of these different sets of numbers are equivalent even though square, cubes, and fourths are subsets of the set of natural numbers. In other words, they share the same cardinality.

Now it is possible to demonstrate the difference between ordinality and cardinality in finite sets as well. If we take two subsets of the natural numbers, say, odds and evens, we can make a new set of the union of the two. This maps to the natural numbers and thus allows us to claim equal cardinality. However, since we remain within the context of the finite, if we start with the odds, there will be no way to "reach" the evens.

$[1,3,5,7,9 \ldots 2,4,6,8 \ldots]$

Regardless of any assumptions about the infinite, there would be no determinate "which one" except for the odds since the ordering does not allow access to the evens in a finite number of steps (i.e. the order means that we would never access the evens since the first ellipsis implies a non-terminating sequence). In other words, we will not know where the number " 2 " arises in the sequence.

This problem accentuates the conceptual difference between ordinality and cardinality in order to underline why size and order are distinct structures even

6 For a reconstruction of the "alternative" history of the infinite, involving figures likes Ibn Qurra, please see Mancosu's 2016 essay collection. Paolo Mancosu, "Measuring the size of infinite collections of natural numbers: Was Cantor's theory of infinite number inevitable?”, Abstraction and Infinity, Oxford University Press, Oxford 2015, pp. 116-153. This paper was earlier published as Paolo Mancosu, "Measuring the size of infinite collections of natural numbers: Was Cantor's theory of infinite number inevitable?", Review of Symbolic Logic 2 (4/2009):612-646. For Galileo’s demonstration see Galileo Galilei, Discourses and Mathematical Demonstrations Relating to Two New Sciences, trans. H. Crew and A. de Salvio, Macmillan, New York 1914, pp. 31-37. 
within the finite cases. In non-finite cases, this difference is accentuated following a similar kind of reasoning.

Without giving its demonstration, let us assume that there are non-finite cardinalities and ordinalities. For the cardinal, we designate $\mathrm{x}_{\mathrm{o}}$ as the size of the finite ordinals collected as a set. This is not a finite number because if it were finite, it would be part of the set being measured. It is non-finite. It is transfinite and infinite in technical and common parlance respectively. However, we hesitate in using the term "infinite" because it is significantly different from traditional notions of infinity as the completion of a non-terminating sequence. By "traditional" I mean here the wavering notions of the infinite as either the "potential" infinite of an unending sequence of successive finite terms, or the "number of numbers", the "actual" totality of numbers qua termination of the succession of finite terms. Here, the traditional ideas concerning the infinite is indeed a family of ideas and intuitions that runs the spectrum from the negative imagination of the "very large" but only ever finite, to an infinite totality that, at the pain of contradiction, must invoke a transcendence over numbers themselves. Variations across this spectrum run the gamut in the history of the infinite since antiquity. The origins of set theory offers an alternative. This alternative breaks with the traditional dialectic of the infinite with the one: either the infinite is not one, and therefore a negative entity, or the infinite is a one, and therefore a totality. As neither a potential infinite nor a totality of finite terms, the modern infinite affirms the non-termination of the count and therefore its non-totality.

By the time of the formalization of ZFC, this break with the traditional dialectic of the one and the infinite has already been accomplished by the innovations of Cantor and Dedekind. However, it is clarifying to see its canonical expression in the ZFC axioms. The axiom of infinity in ZFC specifically bars us from this archaic attachment of the infinite to the one.

The axiom of infinity ${ }^{7}$ states:

$$
\exists x(\varnothing \in x \wedge \forall y \in x(S(y) \in x))
$$

The axiom states that there is an infinite set in the sense that there is a set with $\emptyset$ as its element, and also the successor of that set which is the union of that set

7 Kenneth Kunen, Set Theory, revised edition, College Publications, London 2011, p. 17. 
and its subset. Therefore, there is at least one infinite (or non-finite) set in the sense that it collects the set of the null set and all its successors. This expresses infinity neither as termination nor even transcendence of a non-terminating sequence. Rather it expresses a limit. Regardless of the size of the set offered, there is a set to which it and its successor belongs.

While being one of two of the ZFC axioms that assert the existence of a particular kind of set, the other one being the axiom of the void or null set, it does not assert that the infinite set terminates some non-terminating sequence (i.e. there is no "last" finite number). In the ZFC axiom of infinity, there is no "last one" that counts the series for the series to be measured. In fact, the very existence of a "last one" indicates a finite, rather than an infinite set according to this very axiom.

Traditionally, the infinite could only be defined against the backdrop of its relationship to the "one". This dialectic of the one and the infinite pushed the traditional notion to polarize between a potential infinite (an indefinite, non-terminating term), and a contradictory notion of a "number of numbers" that counts all the numbers. That is, either a negation of the one-total (hence potential), or the embrace of the one-total (hence contradictory). In this modern ZFC context, this dialectic fails to hold. There is neither a "greatest" finite number that counts all the finite, nor is there a completion of the non-terminating series of finite numbers. That is, it elides both the actual and potential infinity. The cardinal $\kappa_{0}$ is instead a measure of that non-terminating series of finites. Hence, the natural numbers, the squares, cubes, etc., are all measured by the same cardinal. Adding a finite number to the cardinal $\kappa_{0}$ returns the same cardinal, moreover, adding squares, cubes, fourths (though there are some shared numbers), each measuring the cardinal $\kappa_{0}$ also returns the same cardinal. This feature of the cardinal matches up with intuitions of inexhaustibility traditionally associated with the infinite, where the subtraction of a finite from an infinite returns an infinite, and the addition of an infinite returns an infinite. This was Galileo's observation in the text related to the principle noted above:

Salviati. So far as I see we can only infer the totality of all numbers in infinite, that the number of squares is infinite, and that the number of the roots is infinite; neither is the number of squares less than the totality of all the numbers, nor the 
latter greater than the former; and finally that the attributes "equal," "greater," and "less," are not applicable to the infinite, but only to finite quantities. ${ }^{8}$

However, whereas Galileo presented this feature of the infinite as a kind of puzzlement, Cantor and his faction took it up as a positive property of the infinite. Dedekind, the other founder of modern set theoretically based mathematics (arriving at results independently from Cantor), took the Galilean puzzle instead as the positive definition of the infinite.

A system $S$ is said to be infinite when it is similar to a proper part of itself; in the contrary case $\mathrm{S}$ is said to be a finite system [...] My own realm of thoughts, the totality $S$ of things, which can be objects of my thought is infinite. For if $s$ signifies an element of $S$, then the thought $s$, that $s$ can be object of my thought, is itself an element of $S .{ }^{9}$

For Dedekind, the system S was his terminology for a set S, "similar" here means "equal in size". The infinite set he denotes here is a set where the proper subset is equal in size (ie. "similar") to itself. In turn, the set that Dedekind gives as an example is the set of his "thoughts". Since every thought can be thought of, thoughts of thoughts provide a simple way to generate a subset that is equinumerous to the original set. The thought of a thought is a thought which already belongs to the original set. Hence, any set that expresses this same feature can be considered infinite. What is crucial here is that the finite sets are determined negatively only as those that are contrary to this case. It is worth remarking that the finite here is the negative case of the prior definition of the infinite set. The ground has shifted from a constructive move from the finite to the infinite to that of a prior infinite multiplicity. ${ }^{10}$

Let us designate this shifting of ground by naming this kind of measurement as "Cantor-Dedekind measure". This can be contrasted to "Euclidian measure" which designates that proper subsets must always be smaller than the origi-

8 Galileo, Discourses and Mathematical Demonstrations Relating to Two New Sciences, pp. 32-33.

9 Richard Dedekind, Essays on the Theory of Numbers, Dover Publications, Mineola 1963, pp. 63-64.

10 Cf. Alain Badiou, Number and Numbers, trans. R. Mackay, Polity Press, Boston 2008, pp. 38-54. 
nal set. We recognize that Euclidian measure applies for finite sets. The set of odd numbers in a finite set will be strictly less than the original set (composed of elements other than odd numbers) because it is a proper subset. In turn, as Dedekind argues, the property of infinite sets is to have proper subsets that measure up to the original set. ${ }^{11}$

\section{Ordering and transfinitude}

Now, we can shift focus away from measurement and turn to ordering. Ordering or ordinality identifies a very different structure. If given a set, say the empty set, we can always move up in order by counting that set to produce a new set that is a successor of that set. By doing this, we can model the ordering of numbers.

\begin{tabular}{|l|l|}
\hline Number & Von Neumann ordinal \\
\hline $0=\{\}$ & $\varnothing$ \\
\hline $1=\{0\}$ & $\{\varnothing\}$ \\
\hline $2=\{0,1\}$ & $\{\varnothing,\{\varnothing\}\}$ \\
\hline $3=\{0,1,2\}$ & $\{\varnothing,\{\varnothing\},\{\emptyset,\{\varnothing\}\}\}$ \\
\hline$\ldots$ & $\ldots$ \\
\hline
\end{tabular}

The ellipsis at the bottom of the chart indicates that this constructive process of the ordinal "count" is non-terminating and therefore opens up into the transfinite sphere as a well.

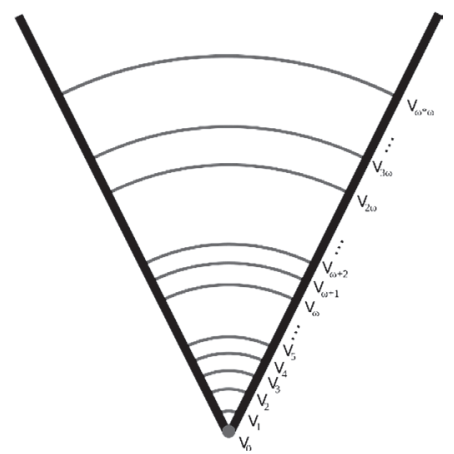

${ }_{11}$ Cantor argues roughly the same thing in Mitteilungen zur Lehre vom Transfiniten. Georg Cantor, “Mitteilungen zur Lehre vom Transfiniten”, Zeitschrift für Philosophie und philosophische Kritik 91 (1887-1888), pp. 81-125, 240-265. Quoted in Mancosu, Abstraction and Infinity, p. 132. 
This hierarchy produced by the ever-widening elements contained in each successor. The "V" shape is due to this. Hence, it is proper to ask the measure of each of these sets that identify the ordinality of the set. In this way, measure and order inform each other. For instance, the first number o contains no elements while the third number contains three elements constructed from previous counts of the set with no elements. However, the difference here is that ordinality requires a correspondence to a well-ordering of the elements, a property independent from measurement. Measurement, whether in the Cantor-Dedekind sense or in the Euclidian sense, requires no appeal to the "least" element. However, for ordinality to make any sense, it must begin with a least element. In the example above drawn from the natural numbers, this least element is o, denoted by the empty set. In other words, in order to "arrive" somewhere in the sequence, it must "begin" somewhere. This difference of structure means that the transfinite ordinals, denoted by $\omega_{n}$, function in a different way to the transfinite cardinals ${\kappa_{n}}_{n}$. The main difference can be reflected by the properties of the traditional infinite mentioned earlier. If we add a finite to the infinite, we get back the infinite. This principle holds in the domain of cardinality. Adding something finite to a set with a transfinite measure does not change its measure. However, adding something finite, like 1, to a transfinite ordinal, can indeed make a difference. The point here is rather simple. The first transfinite ordinality is a border between two orders of succession. Whatever comes before it can be subsumed under it, whatever comes after it moves beyond the border. This is simply what follows from the property of well-ordering between orders. Hence, the addition of an element at the start of the sequence is not the same as the addition of an element at the end of the sequence.

$\mathrm{Z}+\omega=\omega<\omega+\mathrm{Z}$

Though ordinality and cardinality differ in structure, they are not opposed. They are tools that capture different structures in the analysis of sets, especially non-finite sets, which cannot be collapsed into one another. However, insofar as Badiou privileges the count over measure, it is ordinality, not cardinality, that is the guiding structure of his mathematical ontology in L'Être et l'événement. The count is the count of succession rather than that of measurement. Hence, although Badiou accepts the entire machinery of ZFC set theory, the work in L'Être et l'événement nonetheless selects different aspects of the theory to demonstrate certain ontological claims. What is clearly primary is the structure of order, the 
feature of ZFC that grants the "count" of the inconsistent multiplicity entry into the status of being an "ontology". What is secondary is the analysis of their measure, which pertains only to the representations of this primary structure. The key indication of this is the obsolescence of the notion of "infinity" in the text. While we have already hinted at this, we shall turn to make this point more concrete in what follows.

\section{The obsolescence of infinity}

The aim of distinguishing between cardinality and ordinality as structures in the previous section aims at pluralizing the notions of the non-finite (or infinity) that results from the advent of the Cantorian revolution. The goal of this section is to draw out how this implies an obsolescence of the "infinite". This will in turn highlight the pluralization of the "one" in Badiou's ontological project in L'Être et l'événement.

What are the ontological implications for Badiou in distinguishing cardinality and ordinality? First, this distinction between measure and order indicates two different kinds of structure. Cardinality is operated by the one-to-one mapping of one set to another or itself. In non-finite cases, a set is equal in measure to at least one of its proper subsets. Second, ordinality requires a least element, the constructive hierarchy of sets admits to no termination and, more importantly, is indifferent to the properties of traditional infinity. Badiou identifies the structure of ordinals (from finite to the transfinite), and not cardinals, as the arborescent domain of ontology. The parallel but independent structure of cardinality is not rejected but taken up in a secondary role. This point is best viewed through what I shall call the obsolescence of infinity at work in L'Etre et l'événement.

The argument here is simple at first glance. Cardinality and ordinality deal with infinity in very different ways. A non-finite or transfinite cardinal is one where a proper subset is equal measure to the original set. A non-finite or transfinite ordinal marks the border between the finite and non-finite in a non-terminating sequence. However, the addition of an element from the "left" of the sum returns the same ordinal but an addition to the right does not. This is also true of non-terminating sequences in the finite case. For ordinals, the "count" or the ordering of succession determines the order of transfinitude to which a given 
set corresponds. The count, as succession, is entirely indifferent to whether it is progressing in the finite or non-finite domain.

There are two crucial implications here. First, the count is indifferent to the distinction of the finite and non- or trans- finite. The count, in Badiou's sense, is not restricted to the countable (denumerable) sets. This means that such a distinction, between the denumerable and indenumerable is only a secondary analysis made upon the count. We shall examine this later on. The second implication is that the traditional dialectic between the one and the infinite loses all significance in this context. What we get in return is a theory of the "count" that, through "counting-as-one", is neither finite nor infinite in the traditional sense. This installs a reckoning of ontological consistency subtracted from both the notion of the one-consistent as finite and the many-inconsistent as infinite. Badiou's act of "subtraction of the one", as he calls it, is not only a move to privilege the multiple against "the one" but also to thoroughly withdraw the relation of the one and the infinite. We shall later examine what this obsolescence of the infinite means for infinity.

Let us treat the first implication first. The traditional paradoxes of infinity concern the one and the many. The infinite arises in cases where the many cannot be reduced to the one or some rule of the one. Hence, traditionally, the infinite cannot be real because it cannot be reduced to a sum of entities (definite parts) that are actual. More importantly, it cannot be a totality except in a non-quantitative sense. Therefore, appeals to actual infinity from Antiquity until the late modern period tend to be what the Scholastic medievals called "hypercategorematical”. The hypercategorematical may be some ultimate Being (God) or absolute reality that outstrips the discursive resources of the very notion of quantity. Alternatively, to speak of an infinite or infinitesimal quantity in the early modern period, theorists like Leibniz used a "syncategorematical" notion of infinity which corresponded to a "fictional" or "manner of speaking" to designate objectivity to the infinite within a restricted domain of discourse (ie. a differential ratio). This allows for the engagement with the structures available through a commitment to the infinite or infinitesimal without a commitment to its reality. ${ }^{12}$

12 Richard T.W. Arthur has argued for Leibniz's commitment to the actuality of syncategorematical infinities. However, the point here is simply that the syncategorematical use of infinity does not commit us to its actuality. Richard T.W. Arthur, "Leibniz's Syncategorematic 
In brief, the primacy of the one is the reason why the infinite cannot be actual. The infinite is neither reducible to "ones" nor can the "ones" form a totality (a whole) in any quantitative sense. The implication to be drawn here is that the irreducibility of the infinite to the one is what forbids its actuality. In turn, the difference between finite and infinite is simply marked by this property of the quantitative reducibility to the one either as part or totality. The limits of this discourse to the reducibility to the one is precisely what is rejected in L'Etre et l'événement. This is of course what is meant by Badiou's notion of the "subtraction of the one". However, this means the subtraction of the infinite as well. Since the concept of the infinite is grasped as the transcendence from the domain of the one, the subtraction of the one implies the obsolescence of the infinite as well. From this, Badiou's ontological use of the count is also subtracted from the one and the infinite. The count-as-one is therefore the designation of an entity (i.e. a set) that does not fall into the traditional dialectic of the one and the infinite (many). The further implication is that Badiou's count does not respect the border between the quantities made up of ones (the realm of finitude) and the counts of non-finite multiplicities.

This indifference of the count to the finite and non-finite is subject to possible misunderstandings. If the count is understood under the aegis of the traditional relation between the one and the infinite, the distinction between the countable and uncountable (denumerable and indenumerable) quantities would present a challenge to Badiou's project. If this were the case, the count could not be universally or generically applied across finite and non-finite cases. That is, the count would itself be subject to the dialectic of the one and the infinite. Instead what Badiou introduces with the count is the creation of the border between the consistent and the inconsistent. The count is therefore orthogonal to the distinction between the finite and non-finite (i.e. the transfinite) and produces, rather than be produced by, the distinction between the consistent and inconsistent.

Actual Infinite”, in O. Nachtomy, R. Winegar (eds.), Infinity in Early Modern Philosophy, Springer, Cham 2018, pp. 155-179. Richard T.W. Arthur, "Leibniz's syncategorematic infinitesimals”, Archive for History of Exact Sciences 67 (5/2013), pp. 553-593. David Rabouin and Richard T.W. Arthur, "Leibniz's syncategorematic infinitesimals II: their existence, their use and their role in the justification of the differential calculus", Archive for History of Exact Sciences 74 (5/2020), pp. 401-443. 
What this implies is that the count is the border between inconsistency and consistency, rather than the distinction between the finite and non-finite. This fact indicates that the count privileges ordinality as its primary functional role. Just to be explicit, if, for the sake of argument, we take Badiou's count to be cardinal measure, we would have to apply some measure to the inconsistent multiple. This is impossible since the inconsistent multiple cannot be measured unless it is first counted-as-one. As such, the inconsistent multiple would have the cardinality of the void. The void stands as what is "before" any count and therefore any measure. Insofar as the inconsistent is neither finite nor non-finite, this does not identify any relation between the finite and non-finite. ${ }^{13}$

The ordinal structure through which the count functions, on the other hand, operates on succession. For the domain of pure sets, the count, outside of the "first" count of the void, operates only by the recursive counting of the predecessor. Hence, nothing about the inconsistent multiple has to be assumed for the count to function. As such, the inconsistent multiple can be taken as a void or really any other kind of ground suitable for an ur-set. There is hence no need for the assumption of any distinction between the finitary status of the count itself. Further, as the count moves up the hierarchy of successors, the count itself does not distinguish between finite and transfinite. That is, while it is true that $\omega_{0}$ must be handled in a different way than a finite ordinal (i.e. it does not commute), the count itself, the process of succession operates indifferently between the finite and infinite.

From this we must assert the fact that the count-as-one does not distinguish between the finite and infinite. This is crucial because the basis of Badiou's ontological project in the L'Être et l'événement does not begin from finite cases that slowly build towards transfinite cases. The count-as-one does not imply finitude or denumerability for either what is counted or what results from the count. Most importantly, it does not imply the difference between denumerability and indenumerability.

13 It should be noted that Badiou has, in L'Immanence des vérités (2018) introduced another approach to inconsistent multiplicity. Here, he uses the Von Neumann universe of sets (V), which is not itself a set but a class, to handle the relation between inconsistent multiplicity and its relationship to sets. The later work certainly implies a more committed relationship to the positivity to the infinite. I reserve my analysis only to the structure worked out in L'Être et l'événement. Alain Badiou, L'Immanence des vérités, Fayard, Paris 2018. 
This indifference of the count to denumerability and indenumerability of the one lead us to the second implication here, the obsolescence of the dialectic between the one and the infinite. The traditional dialectic rejects any "actual" or "determinate" infinity because it cannot be made a one-totality. In its traditional Pre-Cantorian form, the infinite can only be hypercategorematical, a "one" transcending quantity, or syncategorematical, a stipulated infinity (ie. a fiction) based on a restricted domain. Of course, from this traditional perspective, the post-Cantorian transfinite would not qualify as "infinite". However, the transfinite can be considered "infinite" in the sense that the modern view remains the (very useful) distinction between the denumerable and the indenumerable. Insofar as the cardinal $\kappa_{0}$ and the ordinal $\omega_{0}$ identify the measure and count of the denumerables (respectively), thereby forming the limit between the denumerable and indenumerable, the transfinites can be considered "infinite" in a meaningful way. This is however gained only by correlating the transfinites with some pre-given (and familiar) sequence of numbers (the rationals vs. the reals) and with geometrical properties (e.g., the continuum). These are legitimate applications of ZFC but correlations of this sort are not intrinsic to it. The axiom of infinity describes a limit through the operator of belonging and the principle of succession. Denumerability and non-denumerability are applications of this construction.

The main implication here for the purposes of understanding Badiou's countas-one from the axiom of infinity is that the oneness of any set is granted regardless of its finiteness or non-finiteness. From this, unity and infinity do not form a determinate negation. Transfinitude or the form of non-termination is a robust form of the count-as-one rather than its exception.

Given that the Cantor-Dedekind definition of infinity is encoded in a less quantitative way in the axiom of infinity in ZFC, the analysis of sets moves away from a reliance on number fields as a form of correlation. Hence, the move away from the traditional dialectic between unity and infinity does not take place in the form of a mere rejection of this dialectic but rather as an orthogonal side-stepping of this relation. Within the framework, the one and the infinite are not opposed. Instead, transfinite orders are successive orders of limits which correspond neither to the concept of the one nor the infinite (in the traditional sense). We hence assert the obsolescence of infinity through its replacement by a non-terminable but well-founded hierarchy of successive sets. 
If we take this view retrospectively back into the history of mathematics, it seems that this obsolescence of infinity does not really come at any cost to mathematics. The idea of an absolute infinite never had any real mathematical content (even while sustaining a metaphysical and theological importance). The infinite either remained thoroughly potential or had to be, for the most part, fictionalized. ${ }^{14}$ Instead, where this obsolescence of infinity matters most is in the transformed status of the "one". It is unfortunate that the Cantorian transformation of mathematics is sometimes reduced to a reform of the concept of the infinite and a declaration of its actuality. The "one", through the count qua succession is formally retained. However, the one no longer plays the role of the distinguishing mark of the finite, and thus no longer separates the finite from and non-finite. Instead it plays the role of marking consistent multiplicity across these traditional distinctions. With this subterranean transformation of the "one", we make a more thorough reckoning with this guiding concept of the "subtraction of the one". That is, as Badiou argues elsewhere, the act of subtraction is characterized by the retaining of the positivity of a negation. ${ }^{15}$ In the case of ontology, the subtraction of the one is not a rejection but rather the retaining of its positive role in ontology through the rejection of its traditional dialectic with the infinite. This notion, in parallel to the obsolescence of the infinite does play an important mathematical role. The most crucial of these is the availability of the transfinite ordinals, the use of unity without totality in treating non-finite terms. What this implies is the pluralization of the notion of the "one". We shall examine this further on.

\section{Cardinality as representation}

Before turning to the pluralization of the one, we turn briefly to the positive role played by cardinality in L'Être et l'événement.

If, as we have seen, the ontological project in L'Être et l'événement proceeds through the structure carved out by ordinality, it is also important to indicate what remains of cardinality in the project. As has been emphasized multiple

14 Mancosu points to some exceptions to this mainstream history of the infinite. Cf. Manco$\mathrm{su}$, "Measuring the size of infinite collections of natural numbers: Was Cantor's theory of infinite number inevitable?”, pp. 117-119.

15 See Alain Badiou, "Destruction, Negation, Subtraction - On Pier Paolo Pasolini”, Lacanian Ink, https://www.lacan.com/badpas.htm, accessed Aug. 2020, Los Angeles 2007. 
times, Badiou does not reject cardinality, but treats it as a secondary structure. In fact, for Badiou the structure of cardinality is appropriate for the analysis of representation.

For L'Être et l'événement, presentation and representation differ in their grafting onto two kinds of operations we can make on sets. Presentation is associated with "belonging" $(\in)$. Therefore, whatever is presented in a situation are the elements of the set. ${ }^{16}$ Representation, on the other hand, is associated with "inclusion" (either as subset $\subseteq$ or proper subset $\subset$ ). This inclusion is an operation that recognizes subsets of the situation (situation qua set). Of course, all the elements of a set can be considered as what "is included" in the set but the subsets also involve all possible combination of those elements. This concept of representation involves mere subsets up to the set of all the subsets of the situation. This is the set of all subsets produced by the operation of inclusion is the powerset.

This takes us to Cantor's theorem. The theorem states that given a set A, its powerset $\mathrm{P}(\mathrm{A})$ is of a strictly higher cardinality than $\mathrm{A}$. For finite cases, this is obvious. A set of three elements $\{x, y, z\}$ will have subsets $\{\emptyset\},\{x\},\{y\},\{z\},\{x, y\},\{x-$ $, z\},\{y, z\},\{x, y, z\}$. Hence, a set of three elements, a cardinality of 3 , will have a powerset made up of 8 sets, hence a cardinality of 8 . The basic reckoning here is that the powerset will have a cardinality of $2^{\mathrm{n}}$, where $\mathrm{n}$ is the cardinality of the original set. For non-finite sets, Cantor argued, the same follows. Hence the powerset of all finite numbers, $\kappa_{0}$, is $2^{\mathrm{ko}}$. This powerset of $\mathrm{N}_{0}$ is therefore also of a strictly greater cardinal. The implication here is that there are cardinalities higher than the set of all infinite numbers that can be indicated by the application of the powerset operation $\mathrm{P}(\mathrm{A})$. Furthermore, for any set, finite or nonfinite, the application of the powerset will render a set of strictly higher cardinality.

This feature of the main development in set theory is interpreted by Badiou as the difference between presentation and representation. Badiou refers to the powerset as the "state". One could find reasons to quarrel about this description

16 Although Badiou credits Lyotard for the terminology of "situation", he cites Barwise and Perry on treating a set as a situation. Cf. Jon Barwise, "Situations, Sets and the Axiom of Foundation”, Logic Colloquium 1984, J.B. Paris, A.J. Wilkie, G.M. Wilmers (eds.), Elsevier Science Publishers, Amsterdam 1986, pp. 21-36. Cited in Badiou, Being and Event, p. 484. Thanks to Julian Rohrhuber who carefully pointed out this passage recently. 
of the state but we take it up here only as a technical term. Given a situation, there is a state of the situation. This is the difference between a set and its powerset. This also lines up with presentation and representation. On this account, Badiou offers two analyses. The first is that given the operation of the powerset, the sequence of cardinal numbers is interminable. The second is that the general application of the powerset results in an outstripping, not only of the quantity of the sets involved in the operation, but the outstripping of the scale of measurement. That is, insofar as one moves upwards in cardinality by the powerset of all the sets in that cardinality, each new powerset installs a new scale of quantity (the hierarchy of transfinites) that is irreducible to the "how many" of the lower scale. Here, Badiou notes that, "the natural measuring scale for multiple-presentations is not appropriate for representations. It is not appropriate for them, despite the fact that they are certainly located upon it. The problem is, they are unlocalizable upon it." ${ }^{17}$ The point here is that for non-denumerable sets (non-finite cases) cardinality locates lower sets within them but cannot localize them due to the expansion in cardinality. Reinterpreting Galileo's demonstration, all sets of natural and rational numbers are of the same cardinality $\aleph_{0}$. In the same way, any mapping of transfinite cardinalities to their powersets will result in this same "unlocalizability". The distinctions of a lower dimension are "lost" in the higher dimension. The "state" of a situation, whose constituents are subsets of the situation is therefore always at a higher cardinality than the set of the constituents itself.

The resources of cardinality are employed in L'Être et l'événement. However, they are used in order to address the difference between presentation (governed by belonging), and representation (governed by inclusion). This gap, for Badiou highlights what he calls the "impasse of ontology". This is undergirded by Easton's theorem which roughly states that the cardinality of the powerset $2^{\mathrm{ko}}$ of $\aleph_{0}$ is arbitrarily greater than $\aleph_{0}{ }^{18}$ Essentially, this means that the cardinality of the given powerset of a cardinal $\aleph_{0}$ and greater is any cardinal arbitrarily greater than $\aleph_{0}$. Badiou puts it in the following way:

Badiou, Being and Event, p. 278.

18 A few conditions apply here. Crucially, the set in question is regular (ie., obeying the axiom of regularity/foundation). 
This theorem roughly says the following: given a cardinal $\lambda$, which is either $\omega_{0}$ or a successor cardinal, it is coherent with the Ideas of the multiple to choose, as the value of $|p(\lambda)|$-that is, as quantity for the state whose situation is the multipleany cardinal $\pi$, provided that it is superior to $\lambda$ and that it is a successor cardinal. ${ }^{19}$

Easton's theorem here is a deepening of the Galilean "paradox”. It turns out that the immeasurability of quantities beyond the finite is a general condition that goes beyond the gap between the denumerable and the indenumerable. This is what Badiou will call the "quasi-total errancy". The scales of cardinal infinites interplay with an uncontrollable degree of arbitrariness.

To what degree is this an impasse of ontology then? Badiou argues that,

Consequently, Easton's theorem establishes the quasi-total errancy of the excess of the state over the situation. It is as though, between the structure in which the immediacy of belonging is delivered, and the metastructure which counts as one the parts and regulates the inclusions, a chasm opens, whose filling in depends solely upon a conceptless choice. ${ }^{20}$

The "quasi-total errancy" that Badiou refers to here is precisely the role of cardinality in L'Être et l'événement. That is, given well-ordered sets and consistent multiplicities beyond the finites, the application of cardinality reveals a field of arbitrariness. In other words, all questions of quantity and measure fall into a state of underdetermination where measure is stipulated (chosen) rather than deduced. From this Badiou offers an interpretation of this arbitrariness of the domain of the infinite consistent with our pluralist argument:

Being, as pronounceable, is unfaithful to itself, to the point that it is no longer possible to deduce the value, in infinite extension, of the care put into every presentation in the counting as one of its parts. The un-measure of the state causes an errancy in quantity on the part of the very instance from which we expected-precisely-the guarantee and fixity of situations. The operator of the banishment of the void: we find it here letting the void reappear at the very jointure between itself (the capture of parts) and the situation. That it is necessary to tolerate

\footnotetext{
19 Badiou, Being and Event, p. 279.

20 Ibid., p. 280.
} 
the almost complete arbitrariness of a choice, that quantity, the very paradigm of objectivity, leads to pure subjectivity; such is what I would willingly call the Cantor-Gödel-Cohen-Easton symptom. Ontology unveils in its impasse a point at which thought-unconscious that it is being itself which convokes it therein-has always had to divide itself. ${ }^{21}$

\section{The pluralization of the "one"}

Through examining the difference between cardinality and ordinality, we saw that Badiou's count-as-one is expressed in a transformed concept of the "one". It no longer forms part of the dialectic between the one and the infinite, where the infinite, if it is in any sense actual, must be presented as a totality. What results is a pluralization of the "one".

The argument so far has been the following. The Cantor-Dedekind revolution, while traditionally interpreted as the actualization of infinite sums, should instead be understood as the obsolescence of infinity. The key reason for this is that the "one" as repetition and totality, within this new domain, ceases to play the role of distinguishing between the finite and the infinite. From a cardinality perspective, although denumerability and non-denumerability sustains the traditional border between the finite and infinite as measure, the "infinite" in this sense fails to correspond to the traditional notions of the infinite. Even if we take the infinite to be non-denumerability, we see the problems of the indefinite reproduced via Easton's theorem. Hence the "infinite" in this case, or the realm of the "infinite", fails to be "actual" if we understand this actuality as conditioned, at least, by determination. If we take this question from the perspective of ordinality, we do recover the border between denumerability and indenumerability. The transfinite ordinal as a limit ordinal is logically prior to transfinite cardinalities. ${ }^{22}$ But here, as we have argued, the structure of ordinality does not require

21 Badiou, Being and Event, p. 280.

22 It should be noted here that Cantor saw ordinality and its well-ordering to be more fundamental than cardinality. Georg Cantor, "Über unendliche, lineare Punktmannichfaltigkeiten”, in E. Zermelo (ed.), Gesammelte Abhandlungen mathematischen und philosophischen Inhalts. Mit erläuternden Anmerkungen sowie mit Ergänzungen aus dem Briefwechsel, J. Springer, Berlin 1936; reprinted Olms, Hildesheim 1966, pp. 165-209, p. 169. Although the advent of the cardinal approach to infinity was historically prior, the priority of the ordinal is asserted from a logical perspective. Cf. Hans Niels Jahnke, "Cantor’s cardinal and ordi- 
the infinite in order to stipulate a transfinite limit ordinal. In turn the primacy of ordinality in Badiou's ontological project in L'Être et l'événement, based on the count-as-one ordinality, renders infinity an obsolete concept for ontology. The claim here is to focus instead on the pluralization of the "one".

The pluralization of the notion of the "one" is simply rendered by the notion of the set. The set is a "one" in that it is counted in the ordinal sense. It therefore matters that this count is not confused with measurement. Sets that are built out of the ZFC axioms are placed within a family of branching sets that fulfils the condition of well-ordering. ${ }^{23}$ The basic structure that this ordinality analyses is therefore the structure of succession (i.e. for any set, say a pure set, there is a successor set that counts that set). However, this only gets at the structure and not the existence of sets, the notion of a "one" produced by counting (succession). What then accounts for this parallel structure of existence?

For the project in L'Être et l'événement, Badiou asserts throughout that it is the count that produces the one, eschewing the notion of the "one" as a given. Hence it is the count, an act of counting, that introduces the existence at the basis of the analysis. To stave off misunderstanding, mathematical ontology does not go around counting what exists. Instead it is a rational and logically classical analysis of the result of the act of the count, the unfolding relation between inconsistent multiplicity, what is counted as void, and consistent multiplicity, the aggregation of counts following on the count of the void and its successors (ordinals). What constitutes the grounds of this ontological project theorized by the two "seals" [sceaux] that Badiou introduces in Meditation 14 of L'Etre et l'événement. Badiou's approach pluralizes the "one" treated as the product of the count and thereby concretizes the subtractive aspect of the ontology by revising its traditional basis.

The two "existential seals" occur as limits. The first limit is that which seals off inconsistent multiplicity from consistent multiplicity. Hence, this is the count of the void. Whatever is or is in inconsistent multiplicity is excluded from the

nal infinities: An epistemological and didactic view”, Educational Studies in Mathematics (48/2001), pp. 175-197.

23 Note that while not all sets per se can be well-ordered, in ZFC, Zermelo's theorem shows the equivalence between the axiom of choice and the well-ordering theorem. 
domain of set theoretical entities by inscribing the inconsistent as the void of the consistent structure carved out by sets in their ordinal arrangement. From this, as we have already discussed, sets branch off in ordinals by counting the void. This results in a well-ordered tree of sets grounded in this first seal. Now, the second seal is the limit between the finite ordinals, the first transfinite ordinal, and the transfinite orders that follow after the limit as successors. Since the structure of ordinality is constructed by succession, nothing within this structure offers a distinction between the finite and transfinite. The first tranfinite ordinal is stipulated rather than given from the background structure. This border between the finite and transfinite is thus the limit of the finite series and the start of the transfinite series, a second existential seal.

We have discussed the first seal previously and thus set it aside. The second existential seal has also been briefly addressed. However, it is important here to underline that although, under a traditional reading, this limit ordinal introduces an "actual" infinite in the practice of set theory, what it actually provides, in Badiou's reading, is a further application of the subtraction of the one. Here, what is crucial is that the transfinite ordinal is not a new "one" that counts the finite series in completeness. Rather, it is an inscription of a consistent multiplicity beyond finitude. In this sense, the infinite and the transfinite limit do not coincide. As Badiou argues:

[W]e have not yet defined infinity. A limit ordinal exists; that much is given. Even so, we cannot make the concept of infinity and that of a limit ordinal coincide; consequently, nor can we identify the concept of finitude with that of a successor ordinal. If $\alpha$ is a limit ordinal, then $S(\alpha)$, its successor, is 'larger' than it, since $\alpha \hat{I}$ $S(\alpha)$. This finite successor - if we pose the equation successor=finite - would therefore be larger than its infinite predecessor - if we pose that limit = infinite however, this is unacceptable for thought, and it suppresses the irreversibility of the 'passage to infinity'. If the decision concerning the infinity of natural being does bear upon the limit ordinal, then the definition supported by this decision is necessarily quite different. A further proof that the real, which is to say the obstacle, of thought is rarely that of finding a correct definition; the latter rather follows from the singular and eccentric point at which it became necessary to wager upon sense, even when its direct link to the initial problem was not apparent. 
The law of the hazardous detour thereby summons the subject to a strictly incalculable distance from its object. This is why there is no Method. ${ }^{24}$

Badiou moves on from this to define infinity according to this limit ordinal. Any set where the limit ordinal belongs is infinite, any set belonging to the limit is finite. The ontological significance here is not captured by this distinction. What this second seal indicates is that it replays the distinction between the inconsistent and consistent multiples by treating the finites as the inconsistent (because interminable) multiples. That is, just as nothing within consistent finitude can generate the limit transfinite ordinal, nothing in inconsistent multiplicity can generate the count-as-one of the void. This leads us to the key distinction of our investigation. Here Badiou argues,

In the order of existence the finite is primary, since our initial existent is $\emptyset$, from which we draw $\{\varnothing\}, S\{\varnothing\}$, etc., all of them 'finite'. However, in the order of the concept, the finite is secondary. It is solely under the retroactive effect of the existence of the limit ordinal $\omega_{0}$ that we qualify the sets $\emptyset$, $\{\varnothing\}$, etc., as finite; otherwise, the latter would have no other attribute than that of being existent one-multiples. ${ }^{25}$

What Badiou goes on to develop here, at the end of Meditation 14, is the conceptual effect of the Cantorian revolution. This is the distinction between existence and concept. According to the criterion of the order of existence, there is no relation between the finite and infinite, since, qua ordinals, the sets succeed each other. If $\{\alpha\}$ is finite, so is its successor $S\{\alpha\}$. It is only with the limit ordinal $\omega_{0}$ that the limit between the finite and non-finite is marked. Yet, as Badiou argues, this limit is stipulated, and it is only through this stipulation that the finite and infinite can be distinguished. As Badiou argues here in the passage above, what this reveals is not so much a realm beyond the finite, that is, the infinite, but rather a region of the infinite classified as the "finite". This second seal is therefore a repetition of the first seal. Just as the inconsistent multiple can be recognized as inconsistent only after the first seal which designates consistent multiplicity, finitude can only be recognized (and defined) by the designation of the transfinite limit ordinal.

\footnotetext{
24 Badiou, Being and Event, p. 157.

25 Ibid., p. 159.
} 
Badiou's argument here reflects onto our earlier discussion of Dedekind's argument for the non-finite by means of infinite cardinality. In both cases, the finite is treated as a special case for the ordinary "infinitude" of sets. Hence, finitude is only available as a concept with respect to the definition of the infinite. Here, we can quibble with Badiou on the elaboration of this point. Just as the inconsistent multiple is not inconsistent before the forming of consistent multiplicity, the existence of sets and their successors are not finite before the transfinite limit ordinal. It is therefore incorrect to treat the finitude as primary in the order of existence. The crucial difference here is that the count, the structure analyzed by ordinality, is fundamentally indifferent to the distinction between finite and infinite whereas cardinality is fundamentally sensitive to this difference.

Badiou here reinforces the subtraction of the one by drawing out the further implication that the one, the counting-as-one (and forming-into-one) as a result of an act of counting rather than a given. The one, the concept through which being must be analyzed, will always be alien to what is analyzed. This holds in the case of the original count (the count of the void) as well as within consistent multiplicity. That is, the one, within the post-Cantorian context, is released from its association with finitude. The forming-into-one of a set is shown to be finite only in specific contexts but generically non-finite. The limit ordinal demonstrates this in Badiou's interpretation because it shows finitude to itself be a result of a "one" that is formally outside of it and thus capable of "counting" it (qua ordinal) and standing as a limit, a "one" that stands beyond the "ones" that populate finitude.

The new "one" within the post-Cantorian context is thus completely transformed. Another way of saying the same thing is to reaffirm that the dialectical relationship between the one and the infinite no longer holds. The one is no longer distinguished from the infinite but rather part and parcel of it. Just as the infinite is no longer the "beyond" of finitude, the one is no longer the obstacle of infinity.

The reasoning here concerning the one indicates a deep rift between treating ontology by means of ordinality or cardinality. Cardinality relies on the one-toone mapping between sets (or a set to itself) and therefore relies on a traditional notion of the one as a pre-given unit-entity. Here, the infinite or the transfinite involves the equal cardinality of wholes and parts, a suspension of the identity 
between oneness and wholeness (or totality). Hence despite the general coherence in set theory between ordinal and cardinal treatments of the transfinite, Badiou's emphasis on ordinality, via the structure of the count, implies the accommodation of different means of reckoning the measure (i.e. cardinality) of any given set. Hence Badiou's ontological interpretation of ordinality and the limit ordinal implies a pluralization of the one in the sense that the ordinal structure can accommodate different theories of cardinality.

In what follows, we will examine an alternative non-Cantorian treatment of cardinality and revisit the primacy of ordinality in the mathematical ontology of L'Etre et l'événement. What we aim to underline is the pluralization of the "one" as the consequence of the ontological project of L'Être et l'événement.

\section{Numerosity and ordinality}

If we go back to the traditional problems of infinity and the Cantorian revolution, we find that the hallmark of the new "infinity" (in cardinal terms) was the conceptual break with Euclidian measurement. Recall that for Dedekind, an infinitely sized set is one where at least one subset is equal in size to the original set. Cantor's transfinite cardinal also has this property. Also recall that this is logically independent of transfinite ordinality, a question that is relevant but not determinant in questions of measurement per se. However, an alternative approach to infinity has always existed in the sub-currents of mathematics and its philosophical expositors even in the Cantorian age. This is an alternative approach that rejects the relinquishing of traditional Euclidian measurement. Hence, to deepen our inquiry into the pluralization of the one, we should examine the recent resurgence of this Euclidian approach. The refusal to relinquish Euclidian part-whole relations is to express a commitment to the notion of the oneness as wholeness. Its friction with the canonical Cantorian view will allow us to grasp what the pluralism of one offers us.

In what its proponents call "numerosity theory", the Euclidian principle, which maintains that the part is always lesser than the whole, is maintained. Although Bolzano, a senior contemporary of Cantor and Dedekind, developed some features of a concept of the infinite that maintains the Euclidian principle in his 1851 Paradoxes of the Infinite [Paradoxien des Unendlichen], numerosity theory is a 
distinct recent development. ${ }^{26}$ This emerged in a series of papers since 2003 by Benci and Di Nasso around "Numerosities of labelled sets: a new way of counting. ${ }^{27}$ Since 2003, the research program has grown significantly to include several aspects of mathematics including probability. It has also sparked harsh criticism in the philosophy of mathematics notably by Parker. ${ }^{28}$ Significantly, it has also been taken up philosophically and historically by Mancosu, moving beyond the narrow domain of non-standard analysis and set theory.

Numerosity theory, from Benci and Di Nasso's 2003 paper, extends the Euclidian principle standard from finite cases to infinite cases. Citing from Mancosu's reconstruction of the paper ${ }^{29}$, we take Benci and Di Nasso's theory as operating from the maintenance of three principles:

1. if there is a bijection between $A$ and $B$ then $v(A)=v(B)$

2. if $\mathrm{A} \subset \mathrm{B}$ then $\mathrm{v}(\mathrm{A})<\mathrm{v}(\mathrm{B})$

3. If $\mathrm{v}(\mathrm{A})=\mathrm{v}\left(\mathrm{A}^{\prime}\right)$ and $\mathrm{v}(\mathrm{B})=\mathrm{v}\left(\mathrm{B}^{\prime}\right)$ then the corresponding disjoint unions $(\nabla)$ and cartesian products (x) satisfy:

$$
\begin{aligned}
& v(A \nabla B)=v\left(A^{\prime} \nabla B^{\prime}\right) \\
& v(A x B)=v\left(A^{\prime} \times B^{\prime}\right)
\end{aligned}
$$

The first is simply the definition of equivalence from bijection, a standard principle of cardinality. The third is a definition of sums and products in numerosity also standard to sets of this kind. What is distinctive is the second principle that maintains the strictly "lesser than" difference between a set and its proper subset. This is what is under contention.

26 Bernard Bolzano, Paradoxien des Unendlichen, C. H. Reclam, Leipzig 1851.

${ }_{27}$ Vieri Benci and Mauro Di Nasso, "Numerosities of labeled sets: A new way of counting”, Advances in Mathematics, 173(2003), pp. 50-67. Cf. V. Benci, M. Di Nasso, and Marco Forti, "An Aristotelian notion of size", Annals of Pure and Applied Logic, 143 (1-3/2006), pp. 43-53.

28 Matthew Parker, "Set size and part-whole principle", The Review of Symbolic Logic, 6 (2013), pp. 589-612.

29 Mancosu, "Measuring the size of infinite collections of natural numbers: Was Cantor's theory of infinite number inevitable?”, p. 139-145. 
How does numerosity work? Intuitively, what the theory does is provide labels to sets such that subsets can be indexed (labelled) together in finitary way. Mancosu uses, as an illustration, the Italian game of Tombola, a version of Bingo. On a master board with numbers 1-90, pegs are placed when a number is drawn from a well at random. If a called number corresponds to a number on one's own board (an arbitrary rearrangement of 1-90), one pins the number down. The goal is to cover the board with pegs corresponding to the master board. In the middle of the game, how does one check one's progress in the game? There are three options.

First, one could ignore the order of the numbers and simply check if the number of pegs on one's board corresponds with the number of pegs on the master board. This bijective function would be equivalent to the Cantorian approach to cardinality. Second, one could list the pegs in order such that the numbers 1-90 are listed in order. This corresponds to an ordinal approach. Finally, one could label the pegs by designating the numbers by decades: 1-10, 11-20, 21-30, etc. Each label would be partial, given we are in the middle of the game. Under practical circumstances, this would indeed be an odd way to see how one is progressing in the game of Tombola. Nonetheless, what this does is the breakdown of a potentially linear sequence into incomplete partial sums.

The third approach is thus an intuitive image of numerosity. The goal, as Mancosu argues, "is to split a set of objects into boxes each one containing only finitely many objects. The metaphor of putting things in box number 10, 20, and so forth will be captured by the idea of a labelled set. From now on we deal only with countable sets." ${ }^{30}$

A potentially confusing point here is the fact that in a game, each of the boxes (ie. labels), will be incomplete as we have not finished the game. The point is that each of these partial sums constitute an approximation of the cardinality marked by each label. The whole sum will be the sequence of these approximations. The formal definition of numerosity will then rely on how one is to operate these sequences of approximation. Here, I will again cite Mancosu's simplified outline of a "calculus" for numerosities.

\footnotetext{
30 Ibid., p. 139.
} 
Def. 3.1

The sum of two labeled sets $A, B$ is

$\mathrm{A} \oplus \mathrm{B}=\left\langle\mathrm{A} \nabla \mathrm{B}, l_{\mathrm{A}} \oplus l_{\mathrm{B}}>\right.$ where $l_{\mathrm{A}} \oplus l_{\mathrm{B}}(\mathrm{x})=l_{\mathrm{A}}(\mathrm{x})$ if $\mathrm{x}$ is in $\mathrm{A}$ and $l_{\mathrm{B}}(\mathrm{x})$ if $\mathrm{x}$ is in $\mathrm{B}$. [Caveat: take disjoint unions of A and B only]

Def. 3.2

The product of two labeled sets $A, B$ is

$\mathrm{A} \otimes \mathrm{B}=<\mathrm{A} \times \mathrm{B}, l_{\mathrm{A}} \otimes l_{\mathrm{B}}>$ where $l_{\mathrm{A}} \otimes l_{\mathrm{B}}(\mathrm{x}, \mathrm{y})=\max \left\{l_{\mathrm{A}}(\mathrm{x}) ; l_{\mathrm{B}}(\mathrm{y})\right\}$.

Def. 4 Definition of numerosity

A numerosity function for the class $L$ of all countable labeled sets is a map num: L -> N onto a linearly ordered set $<\mathrm{N}, \leq>$ such that the following properties are satisfied $^{31}$ :

(1) If $\# A_{n} \leq \# B_{n}$ for all $n$, then $\operatorname{num}(A) \leq(B)$

(2) $\mathrm{x}<\operatorname{num}(\mathrm{A})$ iff $\mathrm{x}=\operatorname{num}(\mathrm{B})$ for some $\mathrm{B} \subset \mathrm{A}$

(3) If $\operatorname{num}(A)=\operatorname{num}\left(A^{\prime}\right)$ and $\operatorname{num}(B)=\operatorname{num}\left(B^{\prime}\right)$ then

$\operatorname{num}(\mathrm{A} \oplus \mathrm{B})=\operatorname{num}\left(\mathrm{A}^{\prime} \oplus \mathrm{B}^{\prime}\right)$ and similarly for $\otimes$

What is beyond the purposes of this paper is to prove the existence of this numerosity function defined here in Def. 4. Benci and Di Nasso do so in their 2003 paper and they show that it is done with a selective ultrafilter. ${ }^{32}$ The implication that they draw from this is that this fact shows that numerosity theory is independent of ZFC according to its standard interpretation. However, the authors also acknowledge, citing Booth (1969), that selective ultrafilters are compatible with $\mathrm{ZFC}$ (for denumerable sets). The independence of numerosity theory from ZFC is thus left as an open corollary in the 2003 paper.

Regardless of the independence of numerosity from ZFC, it is clear that the numerosity approach to counting is distinct from both the standard cardinal and ordinal approaches. The additional labelling structure imposed onto the count makes good on the part-whole distinction characteristic of finite sums (ie. the

31 Where \# means the approximation of the labelled set.

32 An ultrafilter "filters" some set for subsets containing some element. A selective ultrafilter obtains when the partition of a set into two pieces results in a homogenous set in the ultrafilter. The existence of this kind of ultrafilter is implied by the continuum hypothesis. Cf. Kenneth Kunen, "Ultrafilters and Independent Sets”, Transactions of the American Mathematical Societies, 172 (1972), pp. 229-306. 
Euclidian property). The implication here is that if we put aside ordinality and cardinality, we can analyze at least some transfinite sets in a way that respects this property. This means, in turn, that Cantorian counting is not necessary to our ability to operate with transfinite magnitude or numbers. The impact of Benci and Di Nasso's work is thus an alternative conception of counting that, though independent from ZFC, analyses and measures sets of transfinite magnitude.

It is important to emphasize that though numerosity is an alternative rather than a direct challenge to Cantorian conceptions of the transfinite, it does indeed challenge the philosophical treatment of the infinite received from the standard exposition of set theory. That is, modern infinity (ie. transfinitude) is gained at the expense of the Euclidian principle. More precisely, it challenges Gödel's opinion that Cantor's views on cardinal number was "uniquely" correct. Critics of numerosity like Parker do not dispute Benci and Di Nasso's work on the basis of its cogency but find fault in it as mathematically arbitrary in the sense that numerosity, as an alternative to Cantorian cardinal counting, presents measurements according to a wholly different notion of size. ${ }^{33}$ It turns out that the view, though logically impeccable, presents an alternative whose sole motive is to affirm the Euclidian principle and does little else. Of course, this is not the place to access Parker's criticism or adjudicate the significance of numerosity theory. Yet it is nonetheless pertinent that this alternative challenges the supposed "inevitability" of Cantorian cardinality. ${ }^{34}$ However, this is a historical and philosophical argument, not a mathematical one. Hence, although there were historical detractors, the canonical embrace of the Dedekind-Cantor cardinality approach to measurement of sets was indeed responsible for the emergence of set theory and the transfinite, there was no necessity attached to this development of the history of the infinite. More importantly, Mancosu traces the history of this notion of the infinite back to Ibn Qurra ( $9^{\text {th }}$ century), Grosseteste ( $13^{\text {th }}$ century), and Bolzano ( $19^{\text {th }}$ century). However, our concern here is not the historical, logical, and mathematical legitimacy of this version of the infinite (the non-finite that respects the Euclidian property) and this approach to measurement (numerosity). The motivation here is instead to identify and concretize the notion of the

33 Matthew Parker, "Set size and part-whole principle", p. 20.

34 This argument against inevitability is precisely Mancosu's interest in numerosity theory. Mancosu, "Measuring the size of infinite collections of natural numbers: Was Cantor's theory of infinite number inevitable?”, p. 116. 
pluralization of the "one" through the different ways that set theory has reconfigured the very concept of counting.

Consistent with Parker's criticisms and Mancosu's analysis, it is correct to view numerosity theory as itself the result of Cantorian measurement and the complex maturation of set theory throughout the $20^{\text {th }}$ century. The general historical effect of the delinking of measurement from a pre-given oneness or completeness of what is measured indicates that numerosity bolsters rather than detracts from the impact of the Cantorian reconfiguring of the traditional dialectic between the one and the infinite. Hence, the terms of the dispute between Numerosity and canonical ZFC set theory, does not concern the status of the transfinite but rather how the "one" is to be conceived within it, a dispute that occurs without thereby disturbing the inherent multiplicity of what is counted-as-one. This indicates at least one example of a pluralization of the one adjacent to the counting by ordinals that Badiou takes as canonical in L'Être et l'événement.

The aim in this section was to examine a case for the pluralization of the one. It may have at first seemed that numerosity would undermine some of the fundaments of Badiou's ontology of the count-as-one modelled after ordinality. After all, Badiou's reliance on the standard interpretation of ZFC suggests that the transfinite could only be won at the expense of the relinquishing of the Euclidian principle. This is not the case. There are two ways to parse this issue. The first is that numerosity theory is independent and therefore not contradictory to ZFC, it constitutes a method of measurement that sits alongside cardinality and ordinality which are themselves not equivalent forms of counting. The second, more important, is that it is itself a result of the primacy of the multiple. In this sense, numerosity theory reintroduces the concept of the part-whole into a theoretical domain that no longer sustains the opposition of the one and the infinite.

How does numerosity allow us to reinterpret Badiou's second ontological seal? Insofar as the second ontological seal is based on an interpretation of the first limit ordinal, the theory of numerosity does not affect the status of the order of transfinites. Indeed, numerosity is a theory primarily concerning how the sizes of sets are measured and therefore does not directly imply any critique of ordinality (the order of sets). However it is clear that the Euclidian structure to which numerosity aims at is generated neither by Cantorian cardinality nor ordinality. From this independence or orthogonality, numerosity introduces a new kind of 
"count" reliant on a theoretical aim, the Euclidian property, that engenders a new consistency between the count, multiplicity, and the transfinite. As an alternative theory of measurement, it is not a criticism of traditional set theory but a critique of it. As critique, it demonstrates some limits of the Cantorian theory of measurement, setting forth its own but stops short at the refutation of the standard means of measurement. The emergence of numerosity therefore indicates the further maturation of the pluralization of the "one" in the pluralization of counting. The "one" in this context is therefore neither based on the givenness of the one nor is it subject to the Cantorian "one" of isomorphic mapping.

Within the context of numerosity, Badiou's second seal, the identification of a limit ordinal, is reproduced in a minor way, across the field of the countables. The labels are themselves stipulated limits which have no other justification than being stipulated in a convenient way for the sake of satisfying the Euclidian property. Each partial sum constituting the numerosity function is a diffraction of the limit ordinal akin to that which allowed Badiou to claim the second seal as the transfinite limit ordinal separating the finite and the non-finite (or transfinite) ordinals. The method of numerosity therefore radicalizes the decisionistic or voluntaristic character of the stipulation of the first limit ordinal (the second seal). In other words, the function of the second existential seal is only illustrated by the emergence of the first limit ordinal but not exhausted by it. Numerosity, as a species of the one, proliferates this limit through labelling. It therefore extends the pluralism of the one identified by the second ontological seal.

Ultimately, Badiou's mathematical ontology in L'Être et l'événement relies on the functional relations between the one, the many, and what is beyond, the transfinite or the infinite. However, the content of the ontology does not reduce to the structure exposited by these relations. Hence, the various alternatives presented here about measurement, and therefore the count, are not alternative ontological presentations. They are rather instances of the complex relationship between the inconsistent multiple and the consistent multiple. Numerosity theory provides some friction for the reconsideration of Badiou's project when it is treated from the perspective of the pluralization of the one. Consistent multiplicity is inaugurated by the structure of the ordinals but its measurement, its representation is subject to different modalities of analysis. 


\section{Some concluding remarks}

Though Badiou and his commentators often emphasize the role of the infinite qua transfinite in the project of mathematical ontology, the fundamental structure that guides the ontological project in L'Etre et l'événement is the count. ${ }^{35}$ If we examine the count through its difference with its parallel mathematical structure, the measure, what we find is that the heart of the project consists in the pluralization of the figure of the one. This pluralization subtracts the countas-one from the traditional framework of the dialectic between the one and the infinite and places positively in the role of operating the distinction between inconsistent and consistent multiplicity. The work of mathematical ontology, its analysis and (re)invention of structure can be reduced to the task of bringing inconsistent and consistent multiplicity into new forms of relation. Hence, at the center of this project is the proliferation of new "ones". The one qua the result of the count identifies the "other" (the non-one) within the "one", in other words, the immanence of the inconsistent within the consistent. Hence, the "subtraction of the one" can be reinterpreted as a proliferation of the one.

This leads us to three general conclusions:

First, in the general argument, we have seized on an interpretation of Badiou's decisionism (or voluntarism) about the infinite as stipulation. Clarifying the fundamentality of ordinal structure helps make more sense of the skepticism about method that Badiou introduces in the L'Être et l'événement. The "anti-method" concerning the infinite in L'Être et l'événement can thus be understood as a rational consequence of this distinction between ordinality and cardinality. This allows us to affirm the transfinite as a decision at the same time as limiting this decisionism in L'Être et l'événement to the dimension of representation, or measurement. The ontological basis of this representation instead turns on the relation between consistency and inconsistency, operated by the count, rather than finitude and the infinite.

35 Badiou introduces a different approach to mathematical ontology in L'Immanence des vérités, one that relaxes some of his earlier restrictions concerning the exclusive use of sets. Further work must be done to see the degree to which the new approach conflicts and extends his arguments in L'Être et l'événement. The fundamental difference is the stronger approach to some interpretation of infinity and the engagement with classes over and above sets. The argument in this paper only relates to L'Être et l'événement. 
Second, the figure of the transfinite can be held at a certain distance from the Cantorian measurement. The examination of numerosity theory bolsters the fact that the trade-off between the Euclidian principle and Cantorian cardinality need not also be so. Hence, as Mancosu argues, numerosity reevaluates the apparent "inevitability" of Cantorian measure. The traditional part-whole relation can therefore be regained alongside the expanded powers of extension brought about by the ZFC paradigm. The historical effect of the advent of Cantor's ordinal and cardinal transfinite had the effect of rupturing the traditional relation between the one and the infinite. Hence the "one" in the Post-Cantorian context can be pluralized across cases where the count can be interpreted through the part-whole relation without sacrificing the actuality of the transfinite. The actuality of the transfinite, a stand-in for an "actual" infinite, is not, however, the basis of the work in L'Être et l'événement. Instead, what is crucial is the reconfiguration of the finite as a species of the infinite instead of the infinite as a transcendence of the finite. This concretely asserts that the count as indifferent to finitude and the infinite.

Third, the pluralization of the one here implies its virtuality. For any analysis of the consistent multiple, the pluralism of the one reveals the multiple facets by which any consistent multiple can be brought into relation with inconsistent multiplicity. The virtual force of the one is therefore operative in this pluralism. The one via the count remains the only means by which the inconsistent and consistent multiple are brought into conceptual relation. If the one is not treated as a given, but instead the result of an act of counting, the resultant one of the count is therefore subject to a pluralism. The one is therefore virtual, insofar as it is indispensable for the very possibility of consistent multiplicity but subject to widely differently forms.

What we have only briefly mentioned is that the second part of L'Etre et l'événement, concerning truth, the event, and the subject is possible only by a negation of the first part, concerning mathematical ontology. That is, after having laid out an intricate and complex ontological structure, it is argued that the event is an exception of that structure. Hence Badiou's aim for slowly developing the ontological structure in the first part of the book is precisely to exploit the limitations that can only be grasped if it is sufficiently built up. The discourse on the event and truth is predicated on the fact that set theory is incomplete (it cannot prove its own consistency) and that, in the treatment of this incompleteness, non-con- 
structible sets had to be developed. Hence, the range of sets must include those that fall outside the well-ordered sets constructed by the count of pure sets and the ordinal structure of succession. This is a further case of the pluralization of the count, but further work will be needed to draw out its implication with respect to the present investigation.

Beyond the central claim of this paper, the argument for the plurality and virtuality of the one in Badiou's L'Être et l'événement, the case is made for the ongoing development of mathematical ontology with respect to developments in the field beyond set theory's main achievements ending with Cohen's independence results in the 1960s. It is sometimes argued that Badiou's use of set theory is locked into the state of set theory in the 1988 , when the book was written. ${ }^{36}$ Set theory itself, it seems, no longer represented the cutting edge of mathematics in the $21^{\text {st }}$ century. There is obviously some truth to the notion that the wealth of energy dedicated to set theory in the $20^{\text {th }}$ century has significantly abated since. However, new research continues in this field and the project of mathematical ontology should be revisited on the basis of these developments. The key here is that the attention that we must pay to set theory is only due to its insights into the relation between inconsistent and consistent multiplicity, and not about some special ontological status of sets themselves. In this way, new insights in the practice of set theory can contribute new concepts in mathematical ontology.

\section{References}

Arthur, Richard T.W., “Leibniz's Syncategorematic Actual Infinite”, in O. Nachtomy, R. Winegar (eds.), Infinity in Early Modern Philosophy, Springer, Cham 2018, pp. 155-179.

- "Leibniz's syncategorematic infinitesimals", Archive for History of Exact Sciences 67 (5/2013), pp. 553-593

Badiou, Alain, L'Être et l'Événement, Seuil, Paris 1988

- Being and Event, trans. O. Feltham, Continuum, London 2005

- "New Horizons in Mathematics as a Philosophical Condition: An Interview with Alain Badiou [with Tzuchien Tho]”, Parrhesia 3 (2007), pp. 1-11

- Number and Numbers, trans. R. Mackay, Polity Press, Boston 2008

36 Badiou's L'Immanence des vérités is his own riposte to this criticism, dealing with the latest developments in set theory. This paper offers another kind of response, rooted in L'Etre et l'événement. 
- “Destruction, Negation, Subtraction - On Pier Paolo Pasolini”, Art Center College of Design in Pasadena, 2007, available at: https://www.lacan.com/badpas.htm

Barwise, Jon, "Situations, Sets and the Axiom of Foundation", Logic Colloquium 1984, J.B. Paris, A.J. Wilkie, G.M. Wilmers (eds.), Elsevier Science Publishers, Amsterdam 1986, pp. 21-36

Benci, Vieri, and Di Nasso, Mauro, "Numerosities of labeled sets: A new way of counting”, Advances in Mathematics, 173(2003), pp. 50-67

- Benci, Vieri, Di Nasso, Mauro and Forti, Marco, "An Aristotelian notion of size”, Annals of Pure and Applied Logic, 143 (1-3/2006), pp. 43-53

Bolzano, Bernard, Paradoxien des Unendlichen, C. H. Reclam, Leipzig 1851

- The Paradoxes of the Infinite, trans. D. A.Steele, Routledge, London 1950

Cantor, Georg, “Mitteilungen zur Lehre vom Transfiniten”, Zeitschrift für Philosophie und philosophische Kritik 91 (1887-1888), pp. 81-125, 240-265

- "Über unendliche, lineare Punktmannichfaltigkeiten”, in E. Zermelo (ed.), Gesammelte Abhandlungen mathematischen und philosophischen Inhalts. Mit erläuternden Anmerkungen sowie mit Ergänzungen aus dem Briefwechsel, J. Springer, Berlin 1936; reprinted Olms, Hildesheim 1966, pp. 165-209

Dedekind, Richard, Essays on the Theory of Numbers, Dover Publications, Mineola 1963

Fraser, Zachary Luke (Lucca Fraser), "The Law of the Subject: Alain Badiou, Luitzen Brouwer and the Kripkean Analyses of Forcing and the Heyting Calculus”, Cosmos and History: The Journal of Natural and Social Philosophy 2 (1-2/2006), pp. 94-133

Galileo, Galilei, Discourses and Mathematical Demonstrations Relating to Two New Sciences, trans. Crew and de Salvio, Dover, New York, pp. 31-37

Jahnke, Hans Niels, “Cantor's cardinal and ordinal infinities: An epistemological and didactic view", Educational Studies in Mathematics (48/2001), pp. 175-197

Kunen, Kenneth, "Ultrafilters and Independent Sets", Transactions of the American Mathematical Societies, 172 (1972), pp. 229-306

- Set Theory: An Introduction to Independence Proofs, Elsevier, Amsterdam 1983

Kenneth Kunen, Set Theory, revised edition, College Publications, London 2011

Mancosu, Paolo, "Measuring the size of infinite collections of natural numbers: Was Cantor's theory of infinite number inevitable?”, Abstraction and Infinity, Oxford University Press, Oxford 2015, pp. 116-153

Parker, Matthew, "Set size and part-whole principle”, The Review of Symbolic Logic, 6 (2013), pp. 589-612

Rabouin, David, and Arthur, Richard T. W., “Leibniz's syncategorematic infinitesimals II: their existence, their use and their role in the justification of the differential calculus”, Archive for History of Exact Sciences 74 (5/2020), pp. 401-443 